\title{
Ad Hoc LTE Method for Resilient Smart Grid Communications
}

\author{
Juho Markkula ${ }^{1} \cdot$ Jussi Haapola $^{1}$
}

Published online: 31 October 2017

(C) The Author(s) 2017. This article is an open access publication

\begin{abstract}
LTE network is a good choice for delivering smart grid demand response (DR) traffic. However, LTE connectivity is not pervasively available due to smart meter improper positioning, limited of coverage, or base station software or hardware failures. In this paper, a solution is introduced to overcome issues relating to lack of LTE base station connectivity for user equipment (UE) considered as remote terminal units, i.e. communication interfaces connected to smart meters. The solution is an ad hoc mode for the LTEAdvanced UE. The ad hoc mode is applied to reach a relay node that is the nearest UE with base station connection. DR traffic is delivered between clusters of UEs and a relay node using multi-hop communications. Analytical Markov chain models and a Riverbed Modeler network simulation model are implemented to illustrate the functionalities and the performance when DR traffic is delivered with varying transmission power levels. A detailed physical layer propagation model for device-to-device communications, a static resource allocation in time domain, hybrid automatic repeat request retransmissions, and a capability for a UE to receive uplink transmissions are modeled both analytically and in the simulator. Both the disjoint analysis and simulations show that all packets are successfully transmitted at most with the fourth transmission attempt and the average network delay is low enough to support most of the smart grid DR applications (139.2-546.6 ms).
\end{abstract}

Keywords LTE · D2D communications · Wireless ad hoc networks · Smart grid communications $\cdot$ Demand response

Juho Markkula

juho.markkula@oulu.fi

1 Centre for Wireless Communications (CWC), University of Oulu, P.O. Box 4500, 90014 Oulu, Finland 


\section{Introduction}

Advanced metering infrastructure (AMI) is a combination of smart meters, a communication network, and utility systems [1]. The communication network enables two way communications between the smart meters and the utility system that contains a metering data management system. The main focus of AMI is to provide real-time information and control of household electricity consumption for demand response (DR) applications. A remote terminal unit (RTU), a communication interface connected to a smart meter, transmits information collected by the smart meter to a metering data management system that processes it and delivers feedback. For example, a smart meter could provide the current energy consumption value and a metering data management system could specify the electricity price due to the total consumption of multiple smart meters [2, 3], or a system operator could turn on/off each user's device according to a direct load control program that follows the grid state and the defined load shaping policy [4]. There are various options for the applied wireless communication technology such as long term evolution (LTE), worldwide interoperability for microwave access (WiMAX), IEEE 802.15.4 and IEEE 802.11 [5].

In a public telecommunications infrastructure, base station connectivity may not be available due to inadvertent placement of a UE, network coverage blind spots, or malfunctioning of a base station. RTUs may be installed in a basement or in buildings that have high penetration losses caused by thick walls, metallic windows, or foil-backed insulation, and 3GGP is working to obtain coverage enhancement for this sort of machinetype communication (MTC) devices [6]. In cellular network planning the target may be to obtain the required coverage with the minimum cost. This may cause blind spots in the cellular network coverage, particularly in sparsely populated areas with few base stations. Also faults in network operator equipment or software are not unheard of [7]. These faults may arise from natural phenomena (ice, heavy wind, rain, etc.), blackouts, hardware abrasion, or software errors. The purpose to develop an ad hoc mode for the LTE-Advanced (LTE-A) user equipment (UE) is to increase LTE-A reliability for smart metering requirements [8] and to cope with situations when a directly reachable LTE base station, called evolved universal terrestrial radio access node B (eNB), is not available. In those cases, clusters with multiple UEs that operate as RTUs are formed. In each cluster, one RTU is selected as a cluster head (CLH) to operate as a gateway between the other clusters and the relay that is the nearest UE with eNB connectivity. Thus, the ad hoc mode can be applied in exception situations to guarantee the continuation of smart grid (SG) communication even if in some areas the LTE network coverage is permanently weak or temporarily lost. Figure 1 illustrates a general communications scenario in the proposed ad hoc mode and in the normal mode where an RTU is directly connected with the LTE eNB. The research of this paper is focused on the wireless paths inside a cluster from RTUs to a $\mathrm{CLH}$, and between the CLHs and a relay. The effort is on studying the communication performances in actual user data transmissions without specifying the details of setting up the ad hoc network.

\subsection{Related Work}

In [9], the authors investigated if a public LTE network is suitable for SG automatic meter usage without causing significant hindrance to typical public LTE traffic. Based on the simulation results, regular SG traffic has very little effect on the eNB or the network load. When considering critical emergency events, such as blackout last gasp messaging, with 


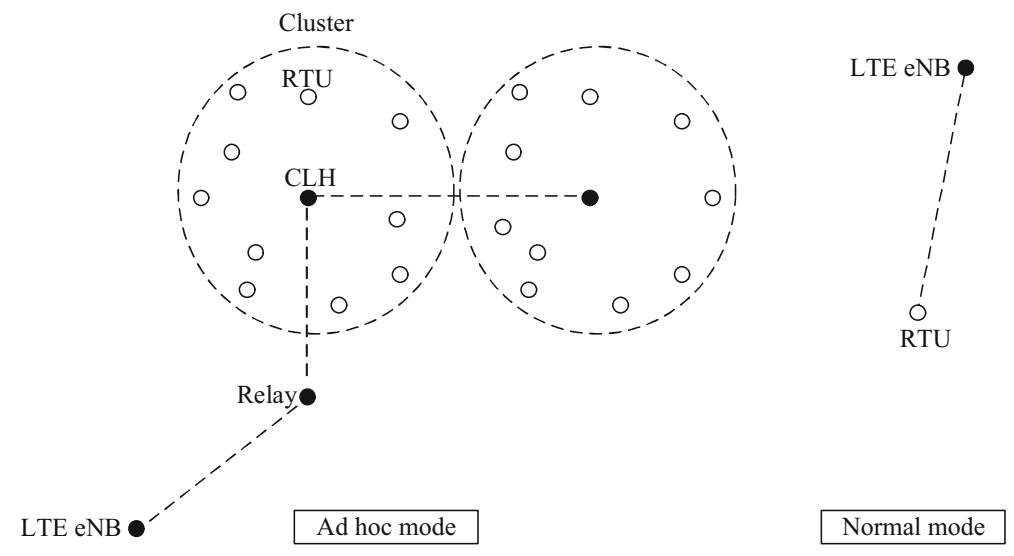

Fig. 1 A general communications scenario in the proposed ad hoc mode (left) and in the normal mode (right)

hundreds of simultaneous packet generations, the network resource allocation capacity was exceeded. Two proposed solutions for mitigating network overloading were effective. The first solution was adding the artificial, $[0,1) \mathrm{s}$ random delay for packet transmissions. The second solution was applying a hybrid sensor-LTE network where RTUs first transmit data to their CLH that contains both an IEEE 802.15.4 and an LTE communication interface. Previously, the authors have simulated SG DR scenarios in an LTE and a hybrid sensorLTE network [10]. There were some differences in performances between these two networks. Applying the hybrid sensor-LTE network seemed to impact less for on the typical public LTE traffic because the antennas of CLHs were located outside on the rooftop height, not inside the houses as RTUs in the LTE network. An uplink scheduling strategy to facilitate the coexistence of smart meters and typical UEs in the LTE network is proposed in [11]. The scheduler considers service differentiation, delay constraints, and channel conditions, and it utilizes relays to decrease the number of direct smart meter connections to the eNB. In [12], the LTE network performance is increased by applying a combination of contention and non-contention based random access procedures for smart meters to establish connections and by delivering SG traffic via tracking area update control signaling to conserve resources at the eNB for typical data transmissions. Limitations of signaling constraints in the random access and control channels are explored with LTE access reservation protocol simulations using machine-to-machine (M2M) traffic [13]. A more efficient procedure in case of M2M connection establishment should be considered by taking into account the features of the actual channels.

There exists quite a lot research about applying device-to-device (D2D) communication between UEs (direct communications) together with the communication via a base station (cellular communications) to improve the communication performance. D2D communications in an LTE-A network with respect to limited interference to cellular traffic are studied in [14]. The decision whether to communicate directly or via the base station (mode selection), spectrum sharing, and power control are essential research targets $[15,16]$.

Multi-hop cellular network (MCN) architecture was initially presented for obtaining a connection to the base station through a relay node [17]. Relaying aims at increasing the capacity and the coverage [18], and a review of the multi-hop relaying strategies for LTE- 
A is presented in [19]. Multi-hop relayed and UE to base station transmissions have different communication performances in terms of transmission rates with distinct radio channel conditions [20]. M2M communication may be performed applying a multi-hop D2D path to avoid burdening the core network [21]. An architectural view of MCN towards LTE-A is evaluated in [22]. LTE for public safety requires D2D communications also without the eNB coverage to guarantee the communication also in disaster areas [23]. D2D functionality for LTE technology is currently under standardization [24-27] and it brings many technical challenges [28, 29]. However, the development is still on a beginning stage, and there are plenty of open research issues in D2D communications [30]. LTE is a promising technology for SG communications, and the D2D enhancement would enable applications that are not possible with the current communications technologies [31]. Multi-hop communications are omitted in the 3GPP Releases 12 and 13 to avoid the use of routing protocols that would increase the complexity $[32,33]$. On the other hand, the SG communications nodes are static that would decrease the complexity of using multihop routing. This paper focuses to on multi-hop M2M/D2D communications in an LTE-A network with SG DR traffic when the eNB coverage suddenly becomes unavailable or is not stable and the traffic is delivered through a relay node.

\subsection{Contribution}

The proposed solution aims at increasing the reliability of a network for SG DR communications that keep the power distribution network stable. When a base station is not available data delivery, with a high reliability, is conducted using D2D propagation path with difficult channel conditions, via multiple hops. Analytical Markov chain models and a Riverbed Modeler (former OPNET modeler suite) network simulation model of the proposed ad hoc LTE method are presented. The system model includes, in addition to the common LTE UE functionalities, a detailed physical layer propagation model for D2D communications, a static resource allocation in time domain, hybrid automatic repeat request (HARQ) retransmissions for D2D traffic, and a capability for a UE to receive uplink transmissions. The analytical and the simulation modeling are performed with SG DR traffic that corresponds to real-time pricing. The network delay and the quantity of retransmissions are estimated with varying transmission power levels. To the best of our knowledge, none of the prior work apply the proposed technical solutions for establishing the ad hoc LTE connectivity and provide such a detailed and realistic performance modelling.

The rest of the paper is organized as follows. Section 2 proposes the system model with technical features and applied key parameters. In Sect. 3 the analytical and the simulation model are presented. The results are presented in Sects. 4 and 5 concludes the paper with some further observations.

\section{System Model}

In development of the proposed ad hoc LTE-A system model various aspects of [24-27] are taken into account. In the ad hoc mode, the UEs are capable of receiving uplink transmissions similarly to the eNB. All data are transmitted and received in the physical uplink shared channel (PUSCH) [34]. A single frequency band is allocated for RTU transmissions in each cluster. RTUs take fixed period turns to occupy a transmission frame 
for transmitting to their CLH. Each CLH has its dedicated transmission band, which it applies for transmitting to one destination node at a time. A star-cluster topology between the RTUs and their CLH is formed, and a static routing between the CLHs is established towards the nearest relay that is connected to a functional eNB. The routing and the frequency band allocations have been performed manually here, but may be considered being done in certain automated ways. For example, CLHs may be selected due to their locations and channel resources may be allocated for them in advance by the eNB for the situations that the connection would be lost. In the case of a connection failure, an ad hoc routing algorithm, e.g. optimized link state routing protocol (OLSR) [35], would establish a route between CLHs towards the nearest relay node, and RTUs would connect to the CLH with the nearest location or with the best channel conditions. Relay candidates would be informed by the eNB to listen possible connection requests when necessary.

The key parameters of the proposal are presented in Table 1. No link adaptation is applied, and a modulation and coding scheme (MCS) index is constantly 11 . The MCS index 11 corresponds to the transport block size index (TBS) number 10 and to the QPSK modulation [34]. The packet payload size is $P L_{p}(100 \mathrm{~B})$ as in real-time pricing in [36], the compressed IP and UDP headers are $H D_{I P \_U D P}(2 \mathrm{~B})$, the LTE header is $H D_{L T E 1}(7 \mathrm{~B})$ for the first segment and $H D_{L T E 2}(5 \mathrm{~B})$ for the next segments. Thus, the total packet size, $S_{\text {packet }}$, is calculated as

$$
S_{\text {packet }}=P L_{p}+H D_{I P \_U D P}+H D_{L T E 1}+\left(n_{s}-1\right) H D_{L T E 2},
$$

where $n_{s}$ is the number of packet segments. When 1 resource block (RB) is allocated, $18 \mathrm{~B}$ may be delivered during one subframe. Thus, a packet must be divided into eight segments and transmitted during eight subframes.

Table 1 Key parameters for ad hoc LTE modeling

\begin{tabular}{ll}
\hline Parameter & Value \\
\hline Center frequency $\left(f_{c}\right)$ : RTU, CLH & $800.09 \mathrm{MHz}, 800.27 \mathrm{MHz}$ \\
Band width $\left(B W_{r x}\right)$ & $180 \mathrm{kHz}(1 \mathrm{resource}$ block $)$ \\
Transmission power $\left(P_{R B}\right)$ & \\
RTU & $2,4,8,20$, and $40 \mathrm{~mW}$ \\
CLH & $12,20,40,60$, and $80 \mathrm{~mW}$ \\
Tx/Rx antenna gain $\left(G_{t x}, G_{r x}\right)$ & $-2 \mathrm{dBi}$ \\
Receiver sensitivity & $-130.5 \mathrm{dBm}$ \\
Antenna height $\left(h_{U E}\right)$ & $1.5 \mathrm{~m}$ \\
Effective antenna height $\left(h_{U E}^{\prime}\right)$ & $0.8 \mathrm{~m}$ \\
Distance $(d)$ : RTU-CLH, CLH-CLH & $100,160 \mathrm{~m}$ \\
Modulation and coding scheme $(\mathrm{MCS})$ & $11(\mathrm{MCS}), 10(\mathrm{TBS}), \mathrm{QPSK}$ \\
HARQ & Max. three retransmissions \\
Number of RTUs $\left(n_{r t u}\right)$, CLHs & 24,2 \\
Packet generation start time of an RTU & Random 0-400 frames $(0-4 \mathrm{~s})$ \\
Packet generation interval of an RTU $\left(g_{p}\right)$ & 400 frames $(4 \mathrm{~s})$ \\
Packet size $\left(S_{p a c k e t}\right)$, payload $\left(P L_{p}\right)$, header & $144,100,44 \mathrm{~B}$ \\
Number of fragments per packet $\left(f_{p}\right)$ & 8 \\
Number of subframes in a frame $\left(s_{f}\right)$ & 10 in total $10 \mathrm{~ms})$ \\
Number of frames between allocations $(m)$ & 24 \\
\hline
\end{tabular}




\subsection{Hybrid Automatic Repeat Request for D2D Traffic}

The LTE HARQ was utilised in the ad hoc mode. Figure 2 depicts the block error ratio (BLER) versus signal-to-noise ratio (SNR) results that were achieved from separate Matlab simulations in the International Mobile Telecommunications Urban Microcell model (IMT UMi) multipath channel of International Telecommunication Union-Radiocommunication Sector (ITU-R) [24, 37]. Each BLER versus SNR curve corresponds to a specific number of transmission attempts (0-3 HARQ retransmissions). Each time a data fragment is retransmitted, the receiver applies chase combining (maximum-ratio combining) [38] to combine the bits from the current and the previous transmission attempt to achieve a better BLER. There is no significant improvement in BLER between the third and the fourth transmission attempts. Thus, the maximum value of three retransmission attempts is sufficient to leverage the full gain of chase combining.

The upper part of Fig. 3 illustrates the HARQ functionality for RTU transmissions. In the first scheduled frame, two fragments are not received correctly (no ACKs are received). Thus, in the following scheduled frame, these data fragments are retransmitted in the corresponding subframes. Up to three retransmissions are allowed for the same fragment before discarding the data. ACKs are applied to inform of correct reception and are assumed to be transmitted in the control channel with $100 \%$ packet delivery ratio.

The lower part of Fig. 3 depicts the HARQ functionality for CLH transmissions. The situation is the same as in the case of an RTU but retransmissions are already performed in the next frame. If a new packet arrives before the previous one is transmitted successfully, the subframes that are not applied for retransmissions may be used for transmitting the new packet.

\subsection{Channel Model}

A pathloss model is defined according to technical documents [24, 39], and the key parameters are presented in Table 1. Propagation of a signal is modeled analytically to calculate average SNR values in line-of-sight (LOS) and non-line-of-sight (NLOS) cases. The probability to apply LOS propagation for a transmission, $P_{r}\{L O S\}$, is presented as [37]

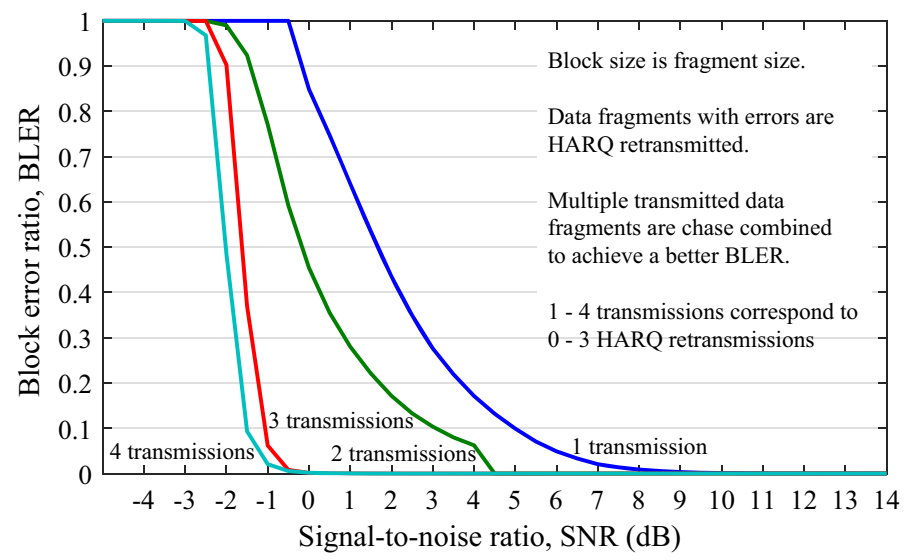

Fig. 2 Block error ratio as a function of signal-to-noise ratio 

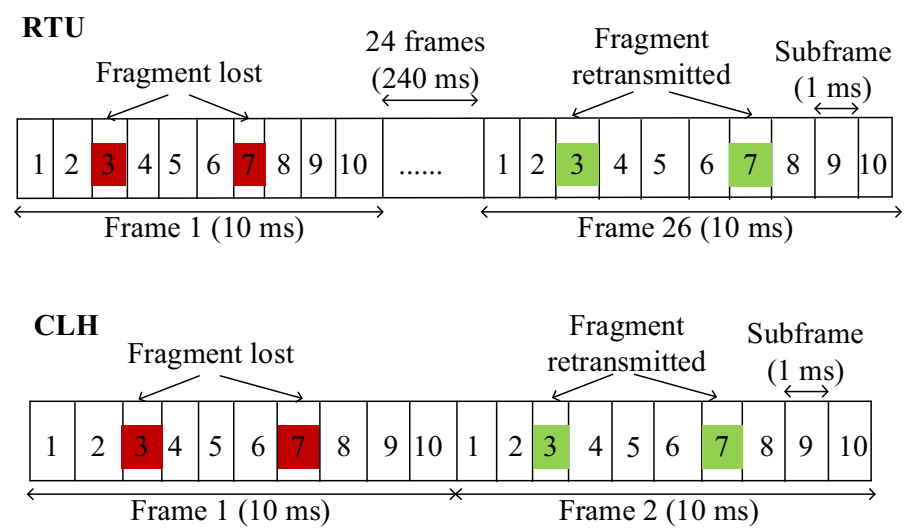

Fig. 3 RTU and CLH retransmission scheduling. Two data fragments are lost. An RTU retransmits in the nearest scheduled frame (upper part), and a CLH retransmits in the next frame (lower part)

$$
P_{r}\{L O S\}=\min (18 / d, 1)[1-\exp (-d / 63)]+\exp (-d / 63),
$$

where $d$ is the distance between two UEs. The probability to apply NLOS propagation is $P_{r}\{N L O S\}=1-P_{r}\{L O S\}$. The pathloss for a LOS case, $P L_{L O S}$, is presented as [24, 39]

$$
P L_{L O S}[\mathrm{~dB}]=40 \log (d)+7.56-34.6 \log \left(h_{U E}^{\prime}\right)+2.7 \log \left(f_{c}\right),
$$

where $h_{U E}^{\prime}$ is the effective antenna height (height of an antenna's radiation center above ground) of a UE, and $f_{c}$ is the center frequency in gigahertz. The pathloss for a NLOS case, $P L_{N L O S}$, is $[24,39]$

$$
\begin{aligned}
P L_{N L O S}[\mathrm{~dB}]= & {\left[44.9-6.55 \log \left(h_{U E}\right)\right] \log (d)+5.83 \log \left(h_{U E}\right)+16.33 } \\
& +26.16 \log \left(f_{c}\right)+O_{N L O S},
\end{aligned}
$$

where $h_{U E}$ is the antenna height of a UE and $O_{N L O S}$ is the NLOS offset $(-5 \mathrm{~dB})$ defined for $\mathrm{D} 2 \mathrm{D}$ communications. The received power, $R P$, with LOS or NLOS propagation is

$$
R P_{\text {LOS } \text { or } N L O S}[\mathrm{~dB}]=P_{R B}[\mathrm{~dB}]+G_{t x}[\mathrm{~dB}]+G_{r x}[\mathrm{~dB}]-P L_{L O S \text { or } N L O S}[\mathrm{~dB}]-P L_{\text {walls }},
$$

where $P_{R B}$ is the allocated transmission power for a single $\mathrm{RB}, G_{t x}$ and $G_{r x}$ are the antenna gains, $P L_{L O S}$ or NLOS is $P L_{L O S}$ or $P L_{N L O S}$, and $P L_{w a l l s}$ is the building entry loss caused by the signal penetration trough building walls (three walls, $18 \mathrm{~dB}$, are applied to model a realistic case) $[40,41]$. The received total noise, $P_{N}$, is

$$
P_{N}[\mathrm{~dB}]=10 \log \left(N_{a c c}+N_{t h}\right),
$$

where $N_{a c c}$ is the accumulated noise power of interfering transmissions ( $0 \mathrm{~W}$, if no concurrent transmissions in the same RB), $N_{t h}$ is the thermal noise presented as [42]

$$
N_{t h}[\mathrm{~W}]=\left(T_{r x}+T_{b k g}\right) B W_{r x} B,
$$

where $T_{r x}$ is the effective receiver temperature (438.4 K) defined as [42]

$$
T_{r x}[\mathrm{~K}]=(N F-1.0) T_{b k g},
$$


where $N F$ is the receiver noise figure (2.512), and $T_{b k g}$ is the effective background temperature $(290 \mathrm{~K}) . B W_{r x}$ is the receiving bandwidth, and $B$ is the Boltzman constant. Finally, the $S N R$ for a LOS or a NLOS case is

$$
S N R_{L O S \text { or } N L O S}[\mathrm{~dB}]=R P_{\text {LOS } \text { or } N L O S}[\mathrm{~dB}]-P_{N}[\mathrm{~dB}] \text {. }
$$

$S N R_{L O S \text { or } N L O S}$ value is applied to select the corresponding BLER value from the specific curve (0-3 HARQ retransmissions) of Fig. 2.

\section{Performance Modeling}

\subsection{Analytical Model}

A mathematical analysis is conducted to illustrate the functionalities and the performance of the developed ad hoc LTE method. Packet transmission details and Markov chains for the two node types, RTU and CLH, are proposed to model a packet delivery.

\subsubsection{Packet Transmission Details}

Further calculations are done to obtain average successful packet transmission probabilities, and average number of transmitted fragments values, with each transmission attempt for the Markov chains. First, $P_{r}\{L O S\}, P_{r}\{N L O S\}$, and $S N R_{L O S \text { or } N L O S}$ values are calculated by (2)-(9) using the parameters of Table 1 . Next, BLER values according to $S N R_{L O S \text { or } N L O S}$ values are obtained form Fig. 2. BLER $\{L O S\}_{-}(n)$ is a BLER in a LOS case, and $B L E R\{N L O S\}_{-}(n)$ is a BLER in a NLOS case, for each number of a transmission attempt $(n \in 1,2,3,4)$. The average BLER between the LOS and NLOS propagation situations is calculated as follows

$$
B L E R_{-}(n)=P_{r}\{L O S\} B L E R\{L O S\}_{-}(n)+P_{r}\{N L O S\} B L E R\{N L O S\}_{-}(n) .
$$

Psuc_( $n)$ correspond to average successful packet transmission probabilities of first, second, and third transmission attempts, $(n \in 1,2,3)$.

$$
\text { Psuc_(n) }=\left[1-B L E R_{-}(n)\right]^{F R_{t r(n)}},
$$

where $F R_{\operatorname{tr}(n)}$ is the average number of transmitted fragments with each transmission attempt, $(n \in 1,2,3)$. The first transmission attempt contains all data fragments of a packet, $F R_{t r(1)}=$ the number of fragments per packet $\left(f_{p}\right)$. A single fragment may be transmitted during a subframe $(1 \mathrm{~ms}) . F R_{t r(n)}$, whose transmission has failed and is retransmitted, is estimated by multiplying the number of previously transmitted fragments with the probability ratio of one fragment error and any possible number of fragment errors. Thus, for the retransmission $(n \in 2,3,4), F R_{\operatorname{tr}(n)}$ can be calculated as

$$
F R_{t r(n)}=F R_{t r(n-1)} \frac{B L E R_{-}(n-1)}{1-P s u c_{-}(n-1)},
$$

where $F R_{t r(n-1)}, B L E R_{-}(n-1)$, and $P s u c_{-}(n-1)$ are values of the previous transmission attempt. Table 2 presents the Psuc_(n), $(n \in 1,2,3)$, values for the communication paths from 24 RTUs to CLH 1, and from CLH 1 to CLH 2, with distinct transmission power levels $\left(P_{R B}\right)$. The packet success probability for the last retransmission attempt $\left(P s u c_{-}(4)\right)$ 
is 1 because all the applied $P_{R B}$ and transmission distance values provided a data delivery without errors $\left(B L E R_{-}(4)=0\right)$. Thus, the $F R_{\operatorname{tr}(4)}$ value is solely applied to estimate a network delay.

\subsubsection{Markov Chain of RTU Transmissions}

The functionality of RTU transmitting packets to a CLH is modeled with the non-saturation Markov chain of Fig. 4. The parameters defined in Table 1, and Psuc_ $(n)$ values presented in Table 2, are applied in the chain. Some additional parameters are further introduced. For each RTU, a frame to transmit is allocated $m$ frames after the RTU's previous allocation has ended. Thus, $m$ is the number of frames between consecutive transmission frame allocations. A Markov chain state illustrates the current operation, and a discrete-time transition based on the results is performed using the step size of $m+1$ frames. Let $P g=(m+1) / g_{p}$ be the probability that a packet is generated during a transmission frame or between consecutive transmission frame allocations, where $g_{p}$ is the packet generation interval of an RTU in frames. The probability of a transmission frame is $P f=1 /(m+1)$. A transition to the blue state [Tr. 1] is performed if a packet is generated at the start or during a transmission frame and all the fragments may be transmitted, or a packet is queued until the start of a transmission frame. The probability that a sufficient number of subframes is left in a transmission frame for a packet transmission is $P s s=\left(s_{f}-f_{p}\right) / s_{f}$, where $s_{f}$ is the number of subframes in a frame. The blue state [Tr. 5] is reached if a packet is generated during a transmission frame and at least one but not all the fragments are able to be transmitted during the remaining subframes. The probability that some subframes are left in a transmission frame but not sufficient to transmit a whole packet is Pnss $=\left(f_{p}-1\right) / s_{f}$. The grey colored state $[Q]$ models the queuing time between a packet generation and a transmission frame. The probability to insert a packet in a queue is $P q u e=1-P f \cdot(P s s+P n s s)$. A transition to the queuing state $[Q]$ is performed if a packet is generated during the last subframe of a transmission frame or during the frames that are not allocated for transmissions of the RTU in question.

The colors blue, green, yellow, and red signify the first, second, third, and fourth transmission attempts, respectively. Consequently, Psuc_ $(n)$ correspond to average successful packet transmission probabilities of first, second, and third transmission attempts, $(n \in 1,2,3) .1-$ Psuc_$_{-}(n)$ is the probability that some fragments of a transmission attempt are not transmitted successfully, $(n \in 1,2,3)$. In the blue state [Tr. 5], a whole packet

Table 2 Average successful packet transmission probabilities of first, second, and third transmission attempts

\begin{tabular}{lllll}
\hline Transmission path & $P_{R B}(\mathrm{~mW})$ & $P_{\text {suc_(1) }}$ & Psuc_(2) & Psuc_(3) \\
\hline 24 RTUs $\Rightarrow$ CLH 1 & 2 & 0.00578 & 0.41736 & 0.99985 \\
$($ distance $=100 \mathrm{~m})$ & 4 & 0.347 & 0.94689 & 1 \\
& 8 & 0.89053 & 1 & 1 \\
& 20 & 0.99802 & 1 & 1 \\
CLH 1 $\Rightarrow$ CLH 2 & 12 & 1 & 1 & 1 \\
(distance $=160 \mathrm{~m})$ & 20 & 0.00001 & 0.04343 & 0.99591 \\
& 40 & 0.03064 & 0.64943 & 0.99997 \\
& 60 & 0.5265 & 1 & 1 \\
& 80 & 0.85779 & 1 & 1 \\
& & 0.94841 & 1 & 1 \\
\hline
\end{tabular}


$P g$ = Probability that a packet is generated during a transmission frame or between consecutive transmission frame allocations.

Pf = Probability of an allocated transmission frame.

Pss = Probability that a sufficient number of subframes is left in a transmission frame for a packet transmission.

Pnss = Probability that some subframes are left in a transmission frame but not sufficient to transmit a whole packet.

$P q u e=$ Probability to insert a packet in a queue.

$P s u c \_(n), \operatorname{Pr}_{t x l}(n), \operatorname{Pr}_{t x 5}(n)=$ Probabilities that fragments of a packet are transmitted successfully.

1- $P s u c_{-}(n), 1-P r_{t x l}(n), P r_{t x 2}(n), P r_{t x 3}(n), P r_{t x 4}(n)=$ Probabilities that at least one fragment of a packet is not transmitted successfully.

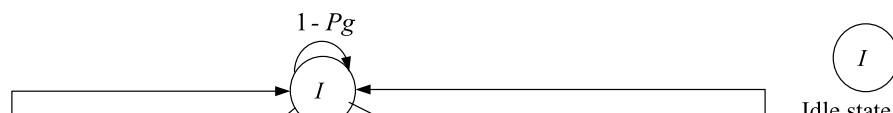

Idle state (No data, previous packet is delivered successfully).
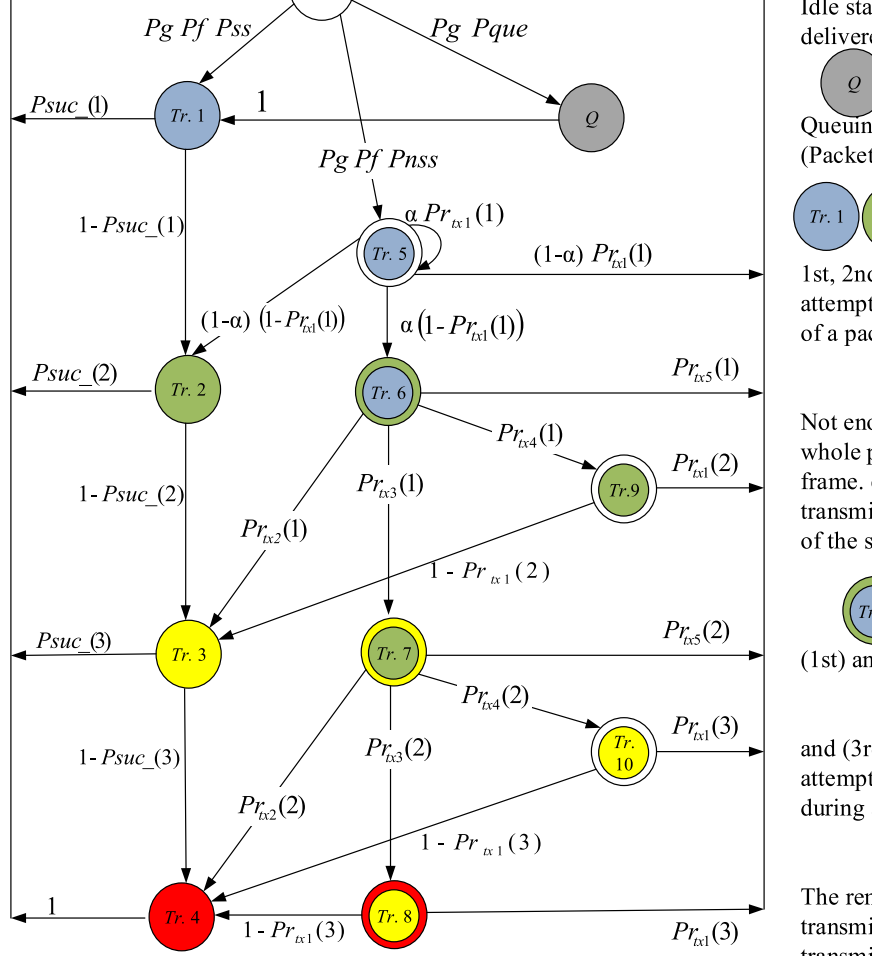

Queuing state

(Packet in a queue).

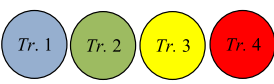

1 st, 2nd, 3rd, and 4th transmission attempts for all the pending fragments of a packet.

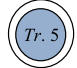

Not enough subframes left to transmit a whole packet in the first transmission frame. $\alpha=$ Probability of the first transmission frame. $1-\alpha=$ Probability of the second transmission frame.
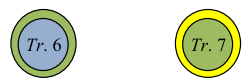

(1st) and [2nd], (2nd) and [3rd],

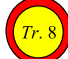

and (3rd) and [4 th] transmission attempts, i.e. two consecutive attempts during a single transmission frame.

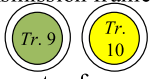

The remainder fragments of a transmission attempt (2nd or 3rd) are transmitted during a transmission frame.

Fig. 4 Non-saturation Markov chain of RTU transmitting packets with maximum three HARQ retransmission (green, yellow, and red states)

cannot be transmitted during a single transmission frame. $\alpha=1 /\left[\operatorname{Pr}_{t x 1}(1)+1\right]$ represents the probability of the first transmission frame, and $1-\alpha$ is the probability of the second transmission frame. Probabilities that some fragments of a packet are transmitted $(n=1)$ or retransmitted $(n \in 2,3)$ correctly in a certain transmission frame are approximated to be $P r_{t x 1}(n)=\sqrt{P s u c_{-}(n)}$ by halving the average number of transmitted fragments $\left(F R_{t r(n)}\right)$ in (11), and $1-\operatorname{Pr}_{t x 1}(n)$ represent the probabilities that at least one fragment is not delivered correctly. The states with two colors, [Tr. 6], [Tr. 7], and [Tr. 8], signify that a packet has two consecutive transmission attempts for separate fragments during a single transmission frame. $\operatorname{Pr}_{t x 2}(n), \operatorname{Pr}_{t x 3}(n)$, and $\operatorname{Pr}_{t x 4}(n)$, are the probabilities that at least one fragment is not 
delivered correctly, $(n \in 1,2)$. $\operatorname{Pr}_{t x 2}(n)$ represent the probabilities that all the delivery failures occur during the latter transmission attempt. $\operatorname{Pr}_{t x 3}(n)$ are the probabilities that delivery failures occur in both transmission attempts. $\operatorname{Pr}_{t x 4}(n)$ represent the probabilities that all the delivery failures occur during the former transmission attempt. $\operatorname{Pr}_{t x 5}(n)$ are the probabilities that all the pending fragments are delivered correctly, $(n \in 1,2)$. In the frames [Tr. 9] and [Tr. 10], the remainder fragments of a retransmission attempt are retransmitted during a transmission frame. The transition back to the state $I$ is performed when all the fragments of a packet are successfully transmitted. Each data packet may contain as many data fragments that can be transmitted in subframes of a frame. The chain (Fig. 4) supports a single packet at a time in the system. Thus, the packet generation interval $\left(g_{p}\right)$ has to be the same or higher than the maximum packet delivery time, i.e. a duration for the four transmissions attempts also containing the waiting between transmission frames.

The state transition probabilities having non-zero values are presented in the Markov chain that is irreducible because there is a sequence of transitions with non-zero probabilities from any state to any other state. It is also aperiodic because none of the states occur periodically. Thus, the Markov chain is ergodic and there exists a steady state solution. A transition matrix that contains the state transition probabilities from each state to every other state is formed, and steady state probabilities are computed numerically. Due to the steady state probabilities, $\pi(\operatorname{Tr} . i)$, of the states $[\operatorname{Tr} .1]-[\operatorname{Tr} .10]$, and the state transition probabilities, $P_{\text {suc }}(I \mid \operatorname{Tr} . i)$, from the states $[\operatorname{Tr} .1]-[\operatorname{Tr} .10]$ to the state $I$, the instantaneous probability that the system is in a given state and a packet is delivered successfully, $P_{\text {suc_st }}(\operatorname{Tr} . i)$, is

$$
P_{\text {suc_st }}(\operatorname{Tr} . i)=P_{\text {suc }}(I \mid \operatorname{Tr} . i) \pi(\operatorname{Tr} . i), i \in\{1,2, \ldots, 10\} .
$$

The probability that a packet is delivered successfully in a given state, $P_{\text {rec }}(\operatorname{Tr} . i)$, is presented as

$$
P_{r e c}(\operatorname{Tr} . i)=\frac{P_{\text {suc_st }}(\operatorname{Tr} . i)}{\sum_{j=1}^{\max (i)} P_{s u c \_s t}(T r . j)} .
$$

The probability that a packet is delivered correctly on a particular transmission attempt, $P_{\text {rec_ta }}(v)$, can be calculated as

$$
P_{r e c \_t a}(v)=\sum_{j \in v} P_{r e c}(T r . j),
$$

where $v \in\{1,2, \ldots, 10\}$ is the sequence of applied states. For instance, the probability that a packet is delivered successfully after the second transmission attempt corresponds to the states [Tr. 2], [Tr. 6], [Tr. 9], and to the $v \in\{2,6,9\}$ values.

The average queuing delay before the first transmission attempt in the blue state [Tr. 1], $D_{\text {que }}(\operatorname{Tr} .1)$, may be calculated as

$$
D_{q u e}(T r .1)=\frac{\left(m s_{f}+1\right) D_{a v r 1}+\left(s_{f}-f_{p}\right) D_{a v r 2}}{m s_{f}+1+s_{f}-f_{p}},
$$

where $D_{\text {avrl }}=0.5\left(m s_{f}+1\right)$ is the average queuing delay affected by the state $[Q]$, and $D_{\text {avr } 2}=0.5$ is the average queuing delay affected by the state [Tr. 1]. The average queuing delay before the first transmission attempt in the state [Tr. 5] is $D_{\text {que }}(\operatorname{Tr} .5)=D_{\text {avr } 2}$. Eventually, the total average queuing delay before the first transmission attempt, $D_{\text {que }}(a v r)$, is presented as 


$$
D_{\text {que }}(\text { avr })=D_{\text {que }}(\operatorname{Tr} .1)(1-\text { PfPnss })+D_{\text {que }}(\operatorname{Tr} .5) \text { PfPnss. }
$$

$D_{p t \_R T U}(\operatorname{Tr} . i)$ is the average delay from the first transmission attempt to the start of a last transmission attempt in the last applied transmission frame. When a packet is delivered correctly during the first applied transmission frame, $D_{p t \_R T U}(\operatorname{Tr} .1)$ is 0 . The states [Tr. 2], [Tr. 3], and [Tr. 4] may be entered from the multiple states through state transitions. Thus, the ratio of the each entering state transition has to be calculated for defining the $D_{p t \_R T U}(\operatorname{Tr} . i),(i \in 2,3,4)$. The steady state probability ratio that the state $[T r . i]$ is entered from the state $[\operatorname{Tr} . j], P_{s s \_r}(\operatorname{Tr} . i, T r . j)$, is presented as

$$
\begin{aligned}
P_{s{ }_{-} \_}(\operatorname{Tr} . i, \operatorname{Tr} . j)= & \frac{\pi(\operatorname{Tr} . j) P_{s t}(\operatorname{Tr} . i \mid \operatorname{Tr} . j)}{\sum_{k \in l} \pi(\operatorname{Tr} . k) P_{s t}(\operatorname{Tr} . i \mid \operatorname{Tr} . k)}, \\
& i \in\{2,3,4\}, j \in\{1,2,3,5, \ldots, 10\},
\end{aligned}
$$

where $P_{s t}(\operatorname{Tr} . i \mid \operatorname{Tr} . j)$ is the state transition probability from the state $[\operatorname{Tr} . j]$ to the state [Tr. $i]$, and $l$ is the sequence of states that have transitions to the state [Tr. $i], l \in\{1,5\}$ when $i=2, l \in\{2,6,9\}$ when $i=3$, and $l \in\{3,7,8,10\}$ when $i=4$. The ratio of $D_{p t \_R T U}(\operatorname{Tr} . j)$ when the state $[T r . i]$ is entered from the state $[\operatorname{Tr} . j], D_{p t \_} R T U_{-} r(\operatorname{Tr} . i, T r . j)$, can be calculated as

$$
D_{p t \_R T U \_r}(\operatorname{Tr} . i, T r . j)=P_{s s \_r}(\operatorname{Tr} . i, T r . j) D_{p t \_R T U}(\operatorname{Tr} . j) .
$$

Finally, $D_{p t \_R T U}(\operatorname{Tr} . i)$ for the states $(i \in 2,3, \ldots, 10)$ can be calculated as

$$
\begin{array}{r}
D_{p t \_R T U}(\operatorname{Tr} .2)=P_{s s \_r}(\operatorname{Tr} .2, \operatorname{Tr} .1)(m+1) s_{f}+D_{p t \_R T U \_r}(\operatorname{Tr} .2, \operatorname{Tr} .5) \\
D_{p t \_R T U}(\operatorname{Tr} . i)=(m+1) s_{f}+\sum_{j \in l} D_{p t \_R T U \_r}(\operatorname{Tr} . i, \operatorname{Tr} . j), i \in\{3,4\} \\
D_{p t \_R T U}(\operatorname{Tr} . i)=\left(F_{\text {num }}-1\right)(m+1) s_{f}-s_{f}+f_{p} / 2, i \in\{5,6, \ldots, 10\},
\end{array}
$$

where $F_{\text {num }}$ is the number of applied transmission frames. $F_{\text {num }}$ is 2 for the states [Tr. 5] and [Tr. 6]. $F_{\text {num }}$ is 3 for the states [Tr. 7] and [Tr. 9]. $F_{\text {num }}$ is 4 for the states [Tr. 8] and [Tr. $10]$.

The average delay of a final fragment in the last applied transmission frame, i.e. an average position of the last subframe applied for a fragment delivery, $D_{f f}(\operatorname{Tr} . i)$, is presented as

$$
\begin{aligned}
D_{f f}(\operatorname{Tr} . i)= & D_{f f \_n}\left(\left\lceil F R_{t r(n)}\right\rceil\right)\left(F R_{t r(n)}-\left\lfloor F R_{t r(n)}\right\rfloor\right) \\
& +D_{f f \_n}\left(\left\lfloor F R_{t r(n)}\right\rfloor\right)\left(\left\lceil F R_{t r(n)}\right\rceil-F R_{t r(n)}\right),
\end{aligned}
$$

where $n$ is the number of a transmission attempt, $n=2$ when $i \in\{2,6,9\}, n=3$ when $i \in\{3,7,10\}, n=4$ when $i \in\{4,8\},\lceil$ presents the ceiling function that rounds up to the nearest integer, and $\rfloor$ presents the floor function that rounds down to the nearest integer. $D_{f f \_n}(N)$ is the delay calculated with the rounded integer values $(N)$ of the $F R_{t r(n)}$ values, and can be calculated as

$$
D_{f f \_n}(N)=\frac{\sum_{h=N}^{f_{p}} h[(h-1) n C r(N-1)]}{\sum_{h=N}^{f_{p}}(h-1) n C r(N-1)}, N \in\left\{1,2, \ldots, f_{p}\right\},
$$

where $n \mathrm{Cr}$ is the number of combinations function. If a packet is delivered correctly after 
the first transmission attempt $(n=1), D_{f f}(\operatorname{Tr} .1)=f_{p}$ and $D_{f f}(\operatorname{Tr} .5)=f_{p} / 2 . D_{f f}(\operatorname{Tr} . i)$ may have values between 1 and $f_{p}$. Finally, the average network delay in subframes, $D_{\text {net_RTU }}(a v r)$, may be calculated as

$$
D_{n e t \_R T U}(a v r)=D_{q u e}(a v r)+\sum_{i=1}^{10} P_{r e c}(\operatorname{Tr} . i)\left[D_{p t \_R T U}(\operatorname{Tr} . i)+D_{f f}(\operatorname{Tr} . i)\right] .
$$

\subsubsection{Markov Chain of CLH Transmissions}

A CLH relays packets it receives from RTUs to another CLH as modeled with the nonsaturation Markov chain of Fig. 5. Most of the parameters applied in the analysis are defined in Table 1, and a discrete-time transition is performed using the step size of one frame. In addition, $\operatorname{Pr}$ is the probability that a packet is received from an RTU during a frame $\left(n_{r t u} / g_{p}\right)$, where $n_{r t u}$ is the number of RTUs. A CLH is assumed to have dedicated channel resources which it can allocate for its own transmissions. Retransmissions of the data fragments are performed in the same subframe positions, where initially transmitted, of the next frame. The transmission and retransmission probabilities $\left(P_{s u c_{-}}(n)\right)$ are defined by (11) and presented in Table $2,(n \in 1,2,3)$. The probability that a packet is delivered successfully on a given state (transmission attempt), $P_{\text {rec }}(\operatorname{Tr} . i)$, may be calculated from the steady state probabilities by (13) and (14) using $i \in\{1,2,3,4\}$, similarly as previously for the Markov chain of RTU transmissions.

The average delay increase due to the fragments of previous packets in the retransmission buffer is estimated in the following cases $\left(c_{r t}\right)$ : no retransmissions in the buffer, first retransmission, second retransmission, third retransmission, or any possible combination of retransmissions in the buffer (first and second, or first and third, or second and third, or first, second, and third), correspondingly $c_{r t} \in\{1,2, \ldots, 8\}$. The probability of each case, $P_{r t}\left(c_{r t}\right)$, is computed numerically, and the average delay increase, $D_{i n c}(a v r)$, is

$$
D_{i n c}(a v r)=\sum_{c_{r t}=2}^{\max \left(c_{r t}\right)}\left(\frac{P_{r t}\left(c_{r t}\right)}{\sum_{j=1}^{\max \left(c_{r t}\right)} P_{r t}(j)} F R_{i n c\left(c_{r t}\right)}\right),
$$

where $F R_{\text {inc }\left(c_{r t}\right)}$ is the number of fragments of previous packets in the retransmission buffer for each case. Cases, $c_{r t} \in\{2,3,4\}$, correspond to $F R_{t r(n)}$ values with $n \in\{2,3,4\}$. Consequently, $F R_{\operatorname{inc}(5)}=F R_{\operatorname{tr}(2)}+F R_{\operatorname{tr}(3)}, F R_{\operatorname{inc}(6)}=F R_{\operatorname{tr}(2)}+F R_{\operatorname{tr}(4)}, F R_{\operatorname{inc}(7)}=F R_{\operatorname{tr}(3)}+$ $F R_{t r(4)}$, and $F R_{i n c(8)}=F R_{t r(2)}+F R_{t r(3)}+F R_{t r(4)}$. The average delay from the first transmission attempt to the start of a last transmission attempt in the last applied transmission frame, $D_{p t \_C L H}(\operatorname{Tr} . i)$, is

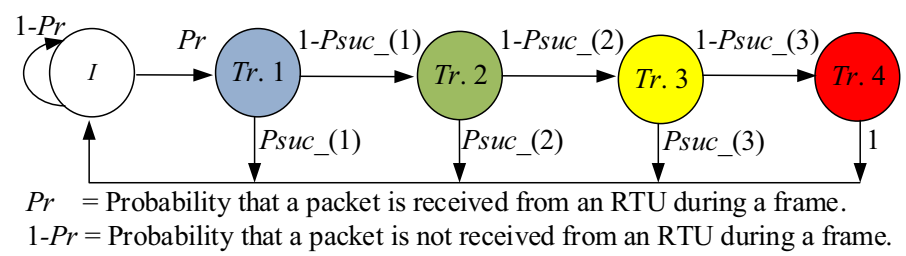

Fig. 5 Non-saturation Markov chain of CLH transmitting packets 


$$
D_{p t \_C L H}(\operatorname{Tr} . i)=(i-1) s_{f}, i \in\{1,2,3,4\} .
$$

The average delay of a final fragment in the last applied transmission frame, $D_{f f}(\operatorname{Tr} . i)$, is defined as previously for RTU transmissions by (21) and (22) using $i \in\{1,2,3,4\}$ and $n \in\{1,2,3,4\}$. Finally, the average network delay in subframes, $D_{\text {net_CLH }}(a v r)$, is

$$
D_{n e t \_C L H}(a v r)=D_{\text {inc }}(a v r)+\sum_{i=1}^{4} P_{r e c}(T r . i)\left[D_{p t \_C L H}(T r . i)+D_{f f}(T r . i)\right] .
$$

\subsection{Simulation Model}

The ad hoc LTE-A network simulation model was developed for Riverbed Modeler that includes the Wireless Suite and LTE simulation toolboxes [43]. Figure 6 presents the LTEA UE node model that was modified to support D2D communications for the nodes (RTU, CLH, and relay), and the scenario where 24 RTUs transmit data to the CLH 1 that relays it to the CLH 2 which also may correspond to a relay node. The DR application applies foursecond reporting intervals. The UE node model includes multiple blocks each containing a communication protocol. A block is implemented as one or multiple process models that

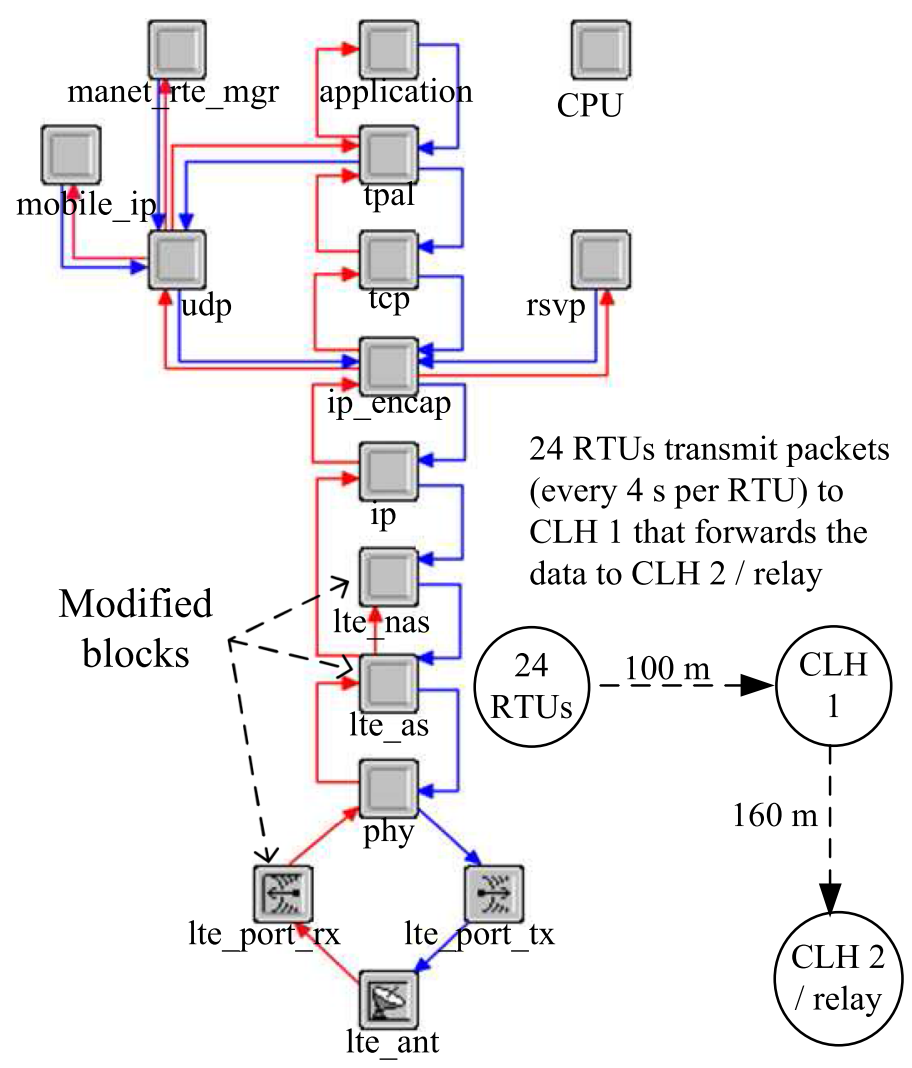

Fig. 6 The LTE-A UE node model modified to provide the ad hoc functionality for the nodes (RTU, CLH, and relay), and the scenario 
contain $\mathrm{C}$ code also including Riverbed Modeler specific functions. The modifications presented in the Sect. 2 were implemented to "lte_as" process model that contains radio resource control (RRC), radio link control (RLC), and media access control (MAC) layer functionalities of a UE. The process model "Ite_nas" was modified to allow a packet delivery to "lte_as" even if there are no active evolved packet system (EPS) bearers because the node is not connected to the eNB. The receiver "lte_port_rx" was modified to be equivalent to the analytical model by implementing the pathloss calculations of (2), (3), (4), (5), and the functionality to fetch a BLER value (Fig. 2) according to the received SNR and the transmission attempt number.

The simulations are performed in packet level and occurring events produce interrupts to distinct process models to perform actions, e.g. a packet is generated in the process model inside the application block. A packet traverses through the blocks between the application and the antenna while some headers are added or removed on a path. The routing between the nodes is set manually due to the adjustable next-hop node address attributes.

\section{Results}

The analytical modeling and the network simulations are carried out in the scenario of Fig. 6 using the parameters of Table 1. The Markov chain of RTU transmissions (Fig. 4) applied the following approximated packet transmission probability values: $P r_{t x 1}(n)=\sqrt{P s u c_{-}(n)}, \quad \operatorname{Pr}_{t x 2}(n)=\sqrt{P s u c_{-}(n)}\left[1-P s u c_{-}(n+1)\right], \quad \operatorname{Pr}_{t x 3}(n)=[1-$ $\left.\sqrt{P s u c_{-}(n)}\right] \quad\left[1-\sqrt{P s u c_{-}(n+1)}\right], \quad \operatorname{Pr}_{t x 4}(n)=\left[1-\sqrt{P s u c_{-}(n)}\right] \sqrt{P s u c_{-}(n+1)}, \quad$ and $\operatorname{Pr}_{t x 5}(n)=\sqrt{P s u c_{-}(n)} P s u c_{-}(n+1)=1-\left[\operatorname{Pr}_{t x 2}(n)+\operatorname{Pr}_{t x 3}(n)+\operatorname{Pr}_{t x 4}(n)\right]$. The results of 100 simulation runs (each $20 \mathrm{~min}$ ) that applied random traffic distributions are averaged. In total, 0.72 million data packets were generated. Table 3 presents the percentage values of correctly received packets with each number of a transmission attempt and with various transmission power levels. The number of a transmission attempt is a sum of the first transmission and at most three retransmissions per hop. The highest percentage values are emphasized with a boldfaced font to show the most probable number of a transmission attempt with each case. The CLH 1 applies a higher transmission power than the RTUs because of longer transmission distance in the DR scenario. It can be seen that the results obtained analytically comply with the simulation results. The difference between the results $(0-1.9 \%)$ is lower with higher transmission powers. All packets are successfully transmitted at most with the fourth transmission attempt (third retransmission).

Table 3 also presents the average network delay values with varying transmission powers. The 24 RTUs share a single RB, but the CLH 1 has its dedicated RB. Thus, a packet delivery between the RTUs and the CLH 1 causes significantly more delay than the delivery between the CLH 1 and the CLH 2. The delay is higher with the lower transmission power because more retransmissions are required. The delay values of the full path (24 RTUs $\Rightarrow$ CLH $1 \Rightarrow$ CLH 2) are from 139.2 to $546.6 \mathrm{~ms}$. The results obtained analytically and with separate simulations are essentially the same (0-4 ms difference) and validate each other's correctness. The results presented the delay in the ad hoc mode before relaying the traffic to the LTE eNB. Our previous work [10] can be applied here to estimate the delay from the relay node to the LTE eNB and to the wired network. With a similar network topology to the one applied in this paper and using the same packet generation interval the SG DR uplink traffic caused approximately $100 \mathrm{~ms}$ network delay in an LTE- 


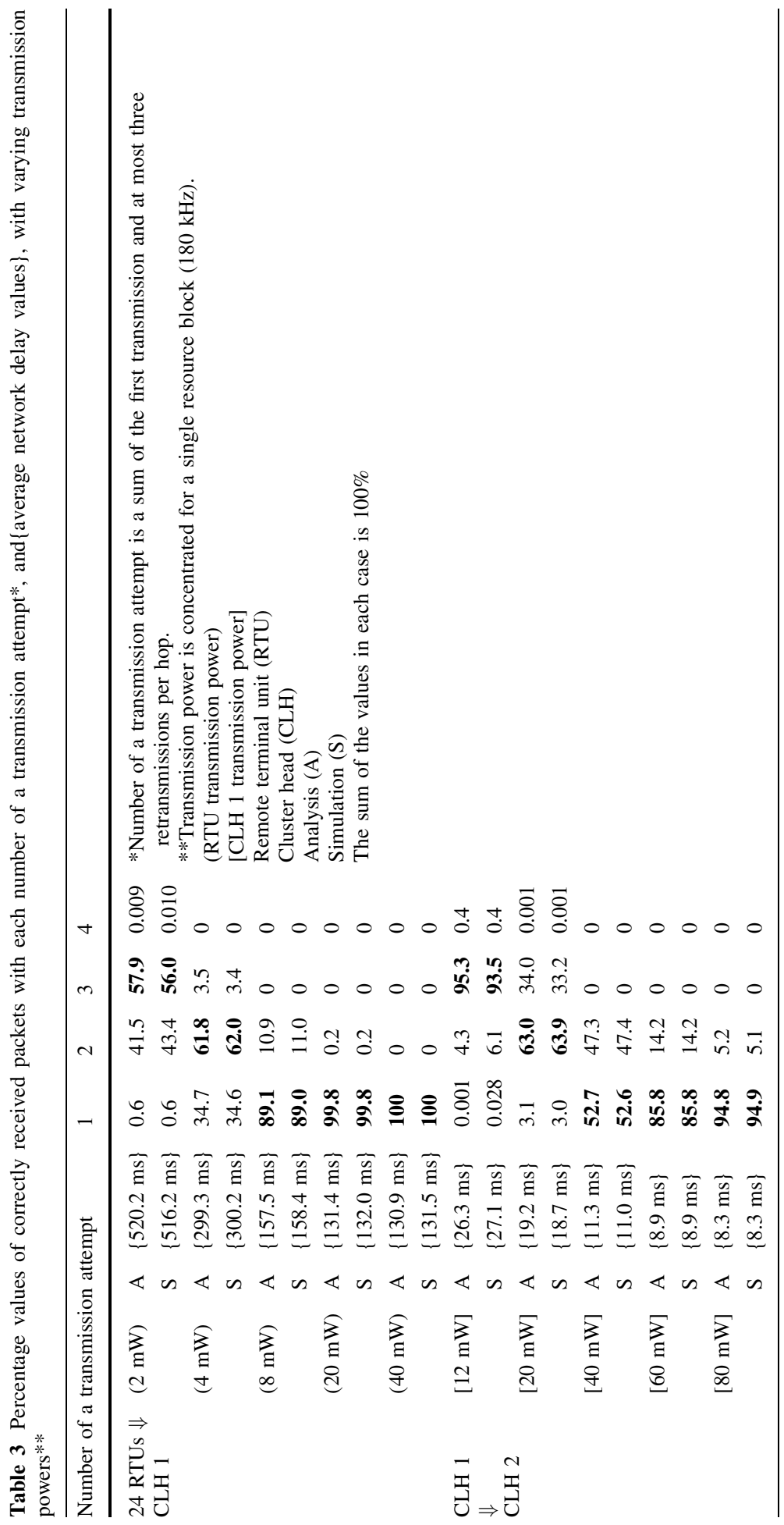




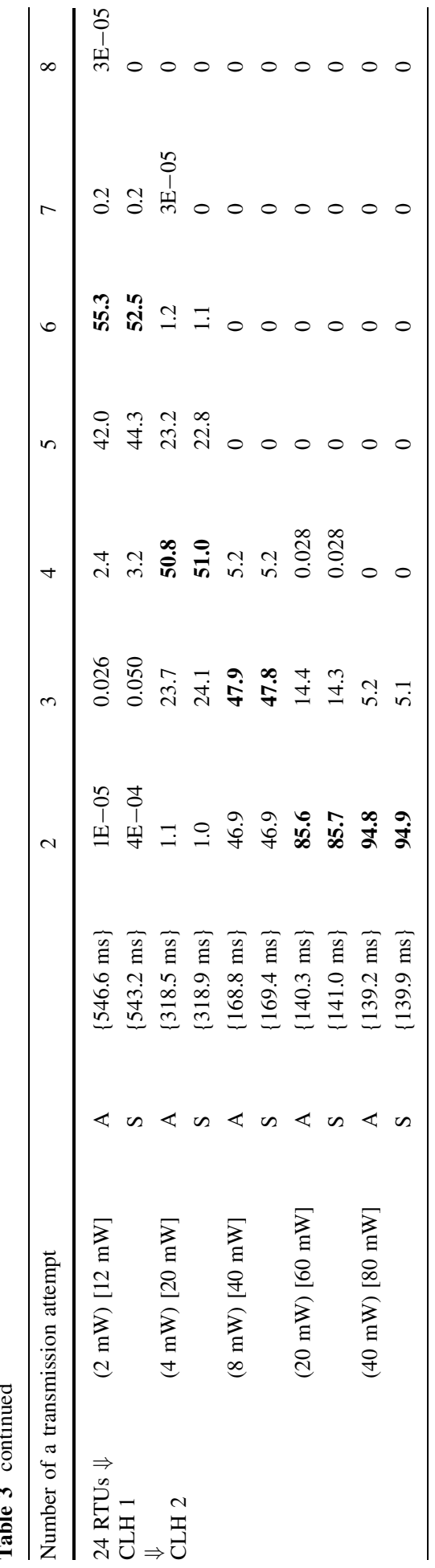


A network that was shared with typical users [10]. The total network delay values range from 239.2 to $646.6 \mathrm{~ms}$ when the ad hoc mode delays and the relay to eNB and to wired network delay estimate, are added up. These delay values are sufficiently low to support most of the SG DR applications [1].

\section{Conclusion and Future Work}

D2D communications is an essential part of the current LTE technology development. This paper proposes an ad hoc mode for an LTE-A UE to overcome issues relating to lack of eNB connectivity for RTUs (communication interfaces connected to smart meters). SG DR communications must be performed by maintaining a packet delivery ratio above $99 \%$. The proposed solution guarantees the SG DR traffic delivery by forming a path through multiple clusters of UEs and a relay (UE with eNB coverage). The LTE-A UE is modified to enable D2D communications with a detailed physical layer propagation model, a static resource allocation in time domain, a HARQ for D2D traffic, and a capability for a UE to receive uplink transmissions.

The scenario contains SG DR communication between RTUs, CLHs, and a relay. The disjoint analytical modeling and the network simulations were performed with various transmission power levels and detailed parameters. With the lower transmission power more retransmissions are required and the average network delay is higher. All packets were successfully transmitted at most with the fourth transmission attempt and the average network delay is low enough to support most of the SG DR applications (139.2-546.6 ms). The analytical and the simulation results are essentially the same and validate each other's correctness.

The future work will consider some enhancements for the current model. A cluster forming method should be defined for setting up the network in the ad hoc mode. Dynamic resource allocation would optimize the usage of the channel resources and decrease the network delay. The suitable multi-hop routing algorithm for the system should be also selected or developed. Extensive network simulations may be performed with the final model using several SG DR applications and a large number of nodes.

Acknowledgements This paper presents work undertaken in the context of the P2P-SMARTEST project, Peer to Peer Smart Energy Distribution Networks (http://www.p2psmartest-h2020.eu/), an Innovation Action funded by the H2020 Programme, contract number 646469. The authors would like to thank Harri Viittala for his support to provide the radio channel simulation results.

Open Access This article is distributed under the terms of the Creative Commons Attribution 4.0 International License (http://creativecommons.org/licenses/by/4.0/), which permits unrestricted use, distribution, and reproduction in any medium, provided you give appropriate credit to the original author(s) and the source, provide a link to the Creative Commons license, and indicate if changes were made.

\section{References}

1. Gungor, V. C., Sahin, D., Kocak, T., Ergut, S., Buccella, C., Cecati, C., et al. (2013). A survey on smart grid potential applications and communication requirements. IEEE Transactions on Industrial Informatics, 9(1), 28-42. https://doi.org/10.1109/TII.2012.2218253.

2. Mohsenian-Rad, A.-H., \& Leon-Garcia, A. (2010). Optimal residential load control with price prediction in real-time electricity pricing environments. IEEE Transactions on Smart Grid, 1(2), 120-133. https://doi.org/10.1109/TSG.2010.2055903. 
3. Ibars, C., Navarro, M., \& Giupponi, L. (2010). Distributed demand management in smart grid with a congestion game. In First IEEE international conference on smart grid communications (pp. 495-500). https://doi.org/10.1109/SMARTGRID.2010.5622091.

4. Molina, A., Gabaldon, A., Fuentes, J. A., \& Alvarez, C. (2003). Implementation and assessment of physically based electrical load models: Application to direct load control residential programs. IEE Proceedings-Generation, Transmission \& Distribution, 150(1), 61-66. https://doi.org/10.1049/ip-gtd: 20020750.

5. Bian, D., Kuzlu, M., Pipattanasomporn, M., \& Rahman, S. (2014). Analysis of communication schemes for advanced metering infrastructure (AMI). In IEEE power and energy society general meeting (pp. 1-5). https://doi.org/10.1109/PESGM.2014.6939562.

6. 3GPP. (2013). 3rd generation partnership project; Technical specification group radio access network; Study on provision of low-cost machine-type communications (MTC) user equipments (UEs) based on LTE [3GPP TR 36.888, Release 12],V12.0.0.

7. Dharmaraja, S., Jindalet, V., \& Varshney, U. (2008). Reliability and survivability analysis for UMTS networks: An analytical approach. IEEE Transactions on Network and Service Management, 5(3), 132-142. https://doi.org/10.1109/TNSM.2009.031101.

8. 5G Infrastructure Association. (2015). White Paper. 5G and Energy, V1.0, Sep. 2015.

9. Markkula, J., \& Haapola, J. (2013). Impact of smart grid traffic peak loads on shared LTE network performance. In IEEE international conference on communications (pp. 4046-4051). https://doi.org/10. 1109/ICC.2013.6655193.

10. Markkula, J., \& Haapola, J. (2013). LTE and hybrid sensor-LTE network performances in smart grid demand response scenarios. In IEEE international conference on smart grid communications (pp. 187-192). https://doi.org/10.1109/SmartGridComm.2013.6687955.

11. Carlesso, M., Antonopoulos A., Granelli, F., \& Verikoukis, C. (2015). Uplink scheduling for smart metering and real-time traffic coexistence in LTE networks. In IEEE international conference on communications (pp. 820-825). https://doi.org/10.1109/ICC.2015.7248423.

12. Karupongsiri, C., Munasinghe, K. S., \& Jamalipour, A. (2016). A novel communication mechanism for Smart Meter packet transmission on LTE networks. In IEEE international conference on smart grid communications (pp. 122-127). https://doi.org/10.1109/SmartGridComm.2016.7778749.

13. Madueño, G. C., Nielsen, J. J., Kim, D. M., Pratas, N. K., Stefanović, Č., \& Popovski, P. (2016). Assessment of LTE wireless access for monitoring of energy distribution in the smart grid. IEEE Journal on Selected Areas in Communications, 34(3), 675-688. https://doi.org/10.1109/JSAC.2016. 2525639.

14. Doppleret, K., Rinne, M., Wijting, C., Ribeiro, C. B., \& Hugl, K. (2009). Device-to-device communication as an underlay to LTE-advanced networks. IEEE Communications Magazine, 47(12), 42-49. https://doi.org/10.1109/MCOM.2009.5350367.

15. Belleschi, M., Fodor, G., \& Abrardo, A. (2011). Performance analysis of a distributed resource allocation scheme for D2D communications. In IEEE GLOBECOM workshops (pp. 358-362). https://doi. org/10.1109/GLOCOMW.2011.6162471.

16. Lin, X., Andrews, J. G., \& Ghosh, A. (2014). Spectrum sharing for device-to-device communication in cellular networks. IEEE Transactions on Wireless Communications, 13(12), 6727-6740. https://doi.org/ 10.1109/TWC.2014.2360202.

17. Lin, Y.-D., \& Hsu, Y.-C. (2000). Multihop cellular: A new architecture for wireless communications. In Nineteenth annual joint conference of the IEEE computer and communications societies (Vol. 3, pp. 1273-1282). https://doi.org/10.1109/INFCOM.2000.832516.

18. Schoenen, R., Halfmann, R., \& Walke, B.-H. (2008). MAC performance of a 3GPP-LTE multihop cellular network. In IEEE international conference on communications (pp. 4819-4824). https://doi.org/ 10.1109/ICC.2008.903.

19. Lo, A., \& Niemegeers, I. (2009). Multi-hop relay architectures for 3GPP LTE-advanced. In IEEE 9th Malaysia international conference on communications (pp. 123-127). https://doi.org/10.1109/MICC. 2009.5431478 .

20. Song, S. H., Almutairi, A. F., \& Letaief, K. B. (2013). Outage-capacity based adaptive relaying in LTEadvanced networks. IEEE Transactions on Wireless Communications, 12(9), 4778-4787. https://doi. org/10.1109/TWC.2013.072513.130217.

21. Rigazzi, G., Chiti, F., Fantacci, R., \& Carlini, C. (2014). Multi-hop D2D networking and resource management scheme for M2M communications over LTE-A systems. In International wireless communications and mobile computing conference (pp. 973-978). https://doi.org/10.1109/IWCMC.2014. 6906487. 
22. Zheng, K., Fan, B., Ma, Z., Liu, G., Shen, X., \& Wang, W. (2009). Multihop cellular networks toward LTE-advanced. IEEE Vehicular Technology Magazine, 4(3), 40-47. https://doi.org/10.1109/MVT.2009. 933474.

23. Doumi, T., Dolan, M. F., Tatesh, S., Casati, A., Tsirtsis, G., Anchan, K., et al. (2013). LTE for public safety networks. IEEE Communications Magazine, 51(2), 106-112. https://doi.org/10.1109/MCOM. 2013.6461193.

24. 3GPP. (2014). 3rd generation partnership project; Technical specification group radio access network; Study on LTE device to device proximity services; Radio aspects [3GPP TR 36.843, Release 12], V12.0.1.

25. Xingqin, L., Andrews, J., Ghosh, A., \& Ratasuk, R. (2014). An overview of 3GPP device-to-device proximity services. IEEE Communications Magazine, 52(4), 40-48. https://doi.org/10.1109/MCOM. 2014.6807945.

26. Ghavimi, F., \& Chen, H.-H. (2014). M2M communications in 3GPP LTE/LTE-A networks: Architectures, service requirements, challenges, and applications. IEEE Communications Surveys \& Tutorials, 17(2), 525-549. https://doi.org/10.1109/COMST.2014.2361626.

27. Nokia Networks. (2014). White Paper. Nokia LTE M2M, optimizing LTE for the internet of things, C401-01085-WP-201409-1-EN.

28. Fodor, G., Dahlman, E., Mildh, G., Parkvall, S., Reider, N., Miklós, G., et al. (2012). Design aspects of network assisted device-to-device communications. IEEE Communications Magazine, 50(3), 170-177. https://doi.org/10.1109/MCOM.2012.6163598.

29. Mumtaz, S., Saidul Huq, K. M., \& Rodriguez, J. (2014). Direct mobile-to-mobile communication: Paradigm for 5G. IEEE Wireless Communications, 21(5), 14-23. https://doi.org/10.1109/MWC.2014. 6940429.

30. Lien, S.-Y., Chien, C.-C., Tseng, F.-M., \& Ho, T.-C. (2016). 3GPP device-to-device communications for beyond 4G cellular networks. IEEE Communications Magazine, 54(3), 29-35. https://doi.org/10. 1109/MCOM.2016.7432168.

31. Kalas, C., \& Alonso-Zarate, J. (2016). Cellular communications for smart grid neighborhood area networks: A survey. IEEE Access, 4, 1469-1493. https://doi.org/10.1109/ACCESS.2016.2551978.

32. 3GPP. (2014). 3rd generation partnership project; Technical specification group radio access network; Study on architecture enhancements to support proximity-based services (ProSe) [3GPP TR 23.703, Release 12], V12.0.0.

33. 3GPP. (2016). Universal Mobile Telecommunications System (UMTS); LTE; Proximity-based services (ProSe); Stage 2 [3GPP TS 23.303, Release 13], V13.4.0.

34. 3GPP. (2014). LTE; Evolved universal terrestrial radio access (E-UTRA); Physical layer procedures; [3GPP TS 36.213, Release 11], V11.6.0 (pp. 43-45 and 100-122).

35. IETF Network Working Group. (2003). Optimized link state routing protocol (OLSR). https://tools.ietf. org/html/rfc3626.

36. Open SG Users Group. (2012). Smart grid networks system requirements specification [Release 5, Final] (pp. 255-256). http://osgug.ucaiug.org.

37. ITU. (2009). Report ITU-R M.2135-1, Guidelines for evaluation of radio interface technologies for IMT-Advanced (pp. 19-23, 33). https://www.itu.int/dms_pub/itu-r/opb/rep/R-REP-M.2135-1-2009PDF-E.pdf.

38. Frenger, P., Parkvall, S., \& Dahlman, E. (2001). Performance comparison of HARQ with chase combining and incremental redundancy for HSDPA. In IEEE 54th vehicular technology conference (Vol. 3, pp. 1829-1833). https://doi.org/10.1109/VTC.2001.956516.

39. Meinilä, J., Kyösti, P., Hentilä, L., Jämsä, T., Suikkanen, E., Kunnari, E., \& Narandžić, M. (2010). D5.3: WINNER+ final channel models, WP5, V1.0 (pp. 74-76). Project document. http://projects.celticinitiative.org/winner+/WINNER+\%20Deliverables/D5.3_v1.0.pdf

40. Anderson, C. R., \& Rappaport, T. S. (2004). In-building wideband partition loss measurements at 2.5 and $60 \mathrm{GHz}$. IEEE Transactions on Wireless Communications, 3(3), 922-928. https://doi.org/10.1109/ TWC.2004.826328.

41. Moldkar, D. (1991). Review on radio propagation into and within buildings. IEE Proceedings on Microwaves, Antennas and Propagation, 138(1), 61-73. https://doi.org/10.1049/ip-h-2.1991.0011.

42. Miller, C. K. S., Daywitt, W. C., \& Arthur, M. G. (1967). Noise standards, measurements, and receiver noise definitions. Proceedings of the IEEE, 55(6), 865-877.

43. Riverbed. Web page. Accessed September 26, 2016, from http://www.riverbed.com. 

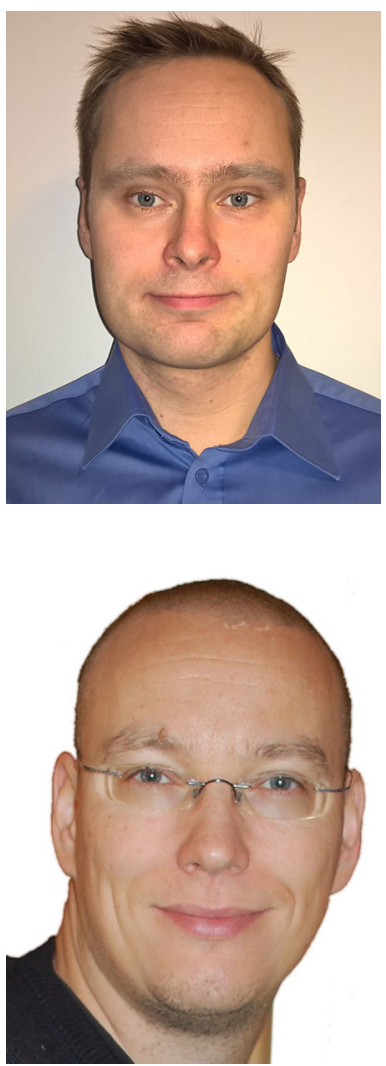

Juho Markkula received the M.Sc degree in Telecommunications from the University of Oulu, Finland, in 2009. Since 2008 he has worked at Centre for Wireless Communications at the University of Oulu, where he currently works as a Doctoral student. He has worked in several research projects concerning military communications system developments. His recent works include research on smart grid communications, especially concerning LTE-A, wireless sensor networks, and low-power wide-area networks.

Jussi Haapola received his M.Sc. and Ph.D. degrees from the University of Oulu, Finland, in 2002 from Physical Sciences and in 2010 from Telecommunications Engineering, respectively. He joined Centre for Wireless Communications at the University of Oulu in 2001, where he currently works as a Postdoctoral researcher. In 2011 he made a Post-doctoral Fellow visit in Tohoku University, Sendai, Japan and between 2012 and 2015 he also worked for University of Oulu Research Institute Japan-CWC-Nippon Ltd as Deputy manager. His recent works include major contributions to two IEEE 802.15 standard amendments and research on smart infrastructure and energy grids. 